\title{
Testing Ranking Statistics for Joint Gravitational Wave and Gamma-Ray Burst Searches
}

\author{
Brandon Piotrzkowski \\ Student (University of Wisconsin-Milwaukee) \\ Milwaukee, WI, USA
}

\begin{abstract}
Joint searches looking for associations between gravitational waves and gamma-ray bursts use ranking statistics to separate real from random coincidences. However, there is no clear choice of which ranking statistics are more useful. We perform a simulated joint search simulating gravitational waves from the LIGO-Virgo-KAGRA (LVK) collaboration and Fermi-GBM gamma-ray candidates from the third operating run of LVK. We find evidence that ranking statistics that include more information on the significance of the individual candidates perform better, although more realistic data is needed to confirm this.
\end{abstract}

\section{Introduction}

Multi-messenger events have become an incredibly important emergent aspect of modern astronomy, typically giving more information about an event than just individual detections. For instance, astronomers have long expected gravitational waves (GW) from binary neutron star (BNS) systems to be accompanied by prompt short gamma-ray bursts (GRB) (Blinnikov et al., 1984, Paczynski, 1986, Eichler et al., 1989). This was confirmed with the joint detection of GW170817 (B. P. Abbott, Abbott, Abbott, Acernese, et al., 2017b a) and GRB 170817A (Goldstein et al., 2017). This BNS-GRB detection was the culmination of the effort of joint searches over the years (Aasi et al., 2014, Singer et al., 2014; B. P. Abbott, Abbott, Abbott, Abernathy, et al., 2017, Urban, 2016, Cho, 2019) and has prompted new searches to look for similar candidates in sub-threshold data (Hamburg et al. |2020; R. Abbott et al. 2020; Stachie et al. 2020, Nitz et al. 2019). Although there have been other potential multi-messenger candidates that involve GWs (Connaughton et al. 2016, Graham et al., 2020), these have been called into question (Greiner et al., 2016, Ashton et al. 2020) leaving us with GW170817-GRB 170817A as the only confident detection. This means that any additional BNS-GRB detections would be invaluable and potentially help constrain the speed of gravity (B. P. Abbott, Abbott, Abbott, Acernese, et al. 2017a), neutron star equation of state (B. Abbott et al., 2018; Coughlin et al., 2019), Hubble constant (Collaboration et al. 2017), and GRB jet models (Farah et al., 2020).

One area of active research for joint searches has been the ranking statistic used, whose function is to rank candidates based on how likely they are to have a real joint origin rather than be randomly associated, an especially difficult task in the sub-threshold regime where more noise events are present. The types of statistics that have been used include modified signal-to-noise ratios (Nitz et al., 2019), false alarm rates (Urban, 2016; Cho, 2019), and other generic ranking statistics (Ashton et al., 2018, Stachie et al., 2020, Piotrzkowski et al., 2021). However, the advantages of each approach has not been well explored, which may mean that searches could be operating at lower sensitivities than otherwise possible.

In this work we test these various statistical methods by using a single simulation between LIGO-Virgo-KAGRA (LVK) GWs and Fermi-GBM GRBs, making both coincidences that resemble real multi-messenger events and those created by random chance. We detail the statistics used in section 2.1 along with the nature of the simulation in section 2.2 . We then discuss how efficiently these methods separated out real from random coincidences in section 3 and then conclude with a discussion in section 4 .

\section{Methods}

2.1. Ranking Statistics Ideally, a ranking statistic should quantify the significance of a joint event, separating real joint events from random coincidences as best as possible. While there exists many approaches, we limited 
ourselves to those currently used or could be used in low-latency searches with the LIGO-Virgo-KAGRA collaboration that operate on the minutes or less time scales, as apposed to higher-latency searches that inject gravitational waves signals in the surrounding data to determine significance ( $R$. Abbott et al., 2020). We considered the odds ratio from Ashton et al. (2018)

$$
\mathcal{O}_{A}=\frac{R_{g w, g r b}^{c} \mathcal{I}_{\Omega}}{\Delta t R_{g w}^{s} R_{g r b}^{s}}
$$

where $R_{a}^{s}$ is the rate of real detections for experiment $a, R_{g w, g r b}^{c}$ is the joint BNS-GRB detection rate, $\Delta t$ is the total time coincidence window, and $\mathcal{I}_{\Omega}$ is the sky map overlap integral. We also considered the odds ratio from Stachie et al. (2020)

$$
\mathcal{O}_{S}=\frac{\mathcal{I}_{\Omega}}{\Delta t\left[1+B_{n / s}\left(x_{g w}\right)+B_{n / s}\left(x_{g r b}\right)+B_{n / s}\left(x_{g w}\right) B_{n / s}\left(x_{g r b}\right)\right]} .
$$

Here $B_{n / s}\left(x_{a}\right)$ is the detection Bayes factor for experiment $a$, weighing whether the data $x_{a}$ is likely from a noise transient $\left(\mathcal{H}_{a}^{n}\right)$ or real signal $\left(\mathcal{H}_{a}^{s}\right)$ as

$$
B_{n / s}\left(x_{a}\right)=\frac{P\left(x_{a} \mid \mathcal{H}_{a}^{n}\right)}{P\left(x_{a} \mid \mathcal{H}_{a}^{s}\right)}
$$

where $P\left(x_{a} \mid \mathcal{H}_{a}^{n}\right)$ is probability of the data $x_{a}$ given that it is noise and $P\left(x_{a} \mid \mathcal{H}_{a}^{p}\right)$ is probability of the data $x_{a}$ given that it is a real signal. We also used the odds ratio from Piotrzkowski et al. (2021)

$$
\mathcal{O}_{P}=\frac{R_{g w, g r b}^{c} \mathcal{I}_{\Omega}}{\Delta t\left[R_{g w}^{s} R_{g r b}^{s}+B_{n / s}\left(x_{g w}\right) R_{g w}^{n} R_{g r b}^{s}+B_{n / s}\left(x_{g r b}\right) R_{g w}^{s} R_{g r b}^{n}+B_{n / s}\left(x_{g w}\right) B_{n / s}\left(x_{g r b}\right) R_{g w}^{n} R_{g r b}^{n}\right]}
$$

where $R_{a}^{n}$ is the rate of noise detections for $a$, and we have omitted some overlap terms and coincident Bayes factors. Note that all these statistics so far have similar derivations but varying levels of approximation, meaning testing these against each other will determine whether additional terms regarding these detection Bayes factors $B_{n / s}\left(x_{a}\right)$ improve sensitivity. Another statistic of interest is the joint false alarm rate (FAR) used by RAVEN (Cho, 2019)

$$
F A R_{c}=F A R_{g w} R_{g r b}^{s} \Delta t / \mathcal{I}_{\Omega}
$$

where $F A R_{g w}$ is the GW false alarm rate.

2.2. Simulation Simulating multi-messenger searches is difficult due to the unique task of trying to combine two disparate experiments in a coherent manner, especially when for real coincidences the underlying parameters must be the same (Ashton et al., 2018). This is still largely an unsolved problem with BNS systems due to the lack of understanding how the underlying models between GWs and GRBs interact. For instance, the lack of strong constraints on the GRB jet model leads to uncertainty in how variations in viewing angle simultaneously affects the detectability of both GWs and GRBs (Farah et al. 2020). Also, other seemingly straightforward relations such as how the neutron star masses affect GRB luminosity remain elusive. For simplicity in the case of real coincidences, we assumed many of the underlying parameters were independent from one another, such as inclination and masses, while we set others, such as timing and sky location, to be same between each experiment. Meanwhile, all the parameters for random coincidence were always set to be independent. This approach is not strictly correct, but is likely the best we can do until we better understand these systems.

In order to test these statistics against each other in the most fair manner possible, we simultaneously computed these for the same set of events. This meant computing several data products to be shared between the various statistics, such as sky maps and detection Bayes factors. We also needed to establish what makes for a simulated real multimessenger detection versus a coincidence from random association. As in Ashton et al. (2018), we define real joint events as having consistent shared parameters (e.g. timing, sky position) and both events being detectable. This latter condition is required in order to relieve the ambiguity with how to handle sub-threshold events, i.e. events that could have real artificial origins but aren't significant enough to be confident detections. Many of these sub-threshold events will inevitably be indistinguishable from noise, since one or more events may be undetectable for a real joint event due to distance/inclination/etc. We explore this by considering two datasets: one that only includes individually significant events (threshold), and another with events that may not be significant individually but their joint significance could be above a joint detection threshold (sub-threshold). We note that the random coincidence datasets will be made up of 
three types of false coincidences: two real candidates that are in reality unrelated, one real and one noise candidate, and two noise candidates. These were proportioned according to the rates we soon define.

Based on these definitions we simulated our various event datasets to be that of the third operating run of LVK (O3). For our GW events, we created a threshold dataset by setting a network signal-to-noise ratio (SNR) (between the Livingston, Hanford, and Virgo detectors) threshold of 12 while our sub-threshold dataset had a threshold of 8. We used tools of ligo. skymap to create a set of true parameters (as opposed to parameters measured by either search which may not be the same). We then used ligo.skymap to create GW localizations using its method of matched filtering, simulating a search done by the LVK. For the GRBs, we drew from given log likelihood ratio (LLR) distributions, with minimum thresholds of 25 for the threshold set and 5 for the sub-threshold set (Stachie et al. 2020; Goldstein et al. 2019). We modeled the GRB sky probability for each event as a Gaussian on the sky as recommended by Fermi-GBM (Connaughton et al., 2015) using gwcelery (GWcelery documentation, 2021). The standard deviation for these Gaussians was given by the LLR (Goldstein et al., 2019) and we then offset from the true source by using the probability sky map generated at this true source as weights. Again, we acknowledge that there should be some correlation between the LLR of the GRB and the true parameters, but at the time of writing this exact relationship isn't well understood. In addition, we chose to conservatively restrict our coincidence dataset to only include GW candidates with viewing angles of less than $25^{\circ}$ (Howell et al., 2019).

We used the rates of a multi-messenger pipeline during $\mathrm{O} 3$, although we note that detection rates are quite uncertain at this time. We set the coincident rate $R_{g w, g r b}^{c}$ to $.58 / y r$, the GW BNS rate $R_{g w}^{s}$ to $5.3 / y r$, the short GRB rate $R_{g w}^{s}$ to $33 / y r$, the rate of noise GW candidates to $1 / h r$, the rate of noise GRB candidates to $1 / 10000 s$, and the total time coincident window $\Delta t$ to $6 s$ (Howell et al., 2019, B. P. Abbott, Abbott, Abbott, Acernese, et al., 2017b; GWcelery documentation, 2021). We ran these simulations for a period of roughly 600 years in order to accumulate enough events to start to represent the underlying distributions.

We calculated the sky map overlap integral $\mathcal{I}_{\Omega}$ (Ashton et al., 2018; Cho, 2019) by

$$
\mathcal{I}_{\Omega}=N_{p i x} \sum_{j=0}^{N_{\text {pix }}} P\left(\Omega_{j} \mid x_{g w}, \mathcal{H}_{g w}^{s}\right) P\left(\Omega_{j} \mid x_{g r b}, \mathcal{H}_{g r b}^{s}\right)
$$

where $P\left(\Omega_{j} \mid x_{a}, \mathcal{H}_{a}^{s}\right)$ is the HEALPix probability for data $x_{a}$ in the sky position $\Omega_{j}$ for pixel $j$, with the total number of pixels $N_{\text {pix }}$, and assuming $a$ has been detected by denoting $\mathcal{H}_{a}^{s}$. We computed our detection Bayes factors similarly to Stachie et al. (2020) by using kernel density estimation (KDE) over intermediary detection statistics, LLR for the GRBs and the detection Bayes factor given in the GW sky maps. To get $F A R_{g w}$ for equation $(5)$, we monotonically mapped from these intermediary detection Bayes factor in the GW sky maps. This approach is not strictly correct since a FAR calculated by a pipeline would emerge from a comparison of the real surrounding background while the detection Bayes factors we used here assumes a Gaussian noise background. Having a more realistic calculation of this $F A R_{g w}$ would give more confidence to the results involving equation (5).

\section{Results}

Having computed our various statistics in our simulated multi-messenger search, we now can analyze their relative performance. We do this by a simple receiver operating characteristic (ROC) analysis, comparing the number of real coincidences (true positives) found given a threshold to the allowed number of random coincidences (false positives). This allows us to test every statistic regardless of their mathematical form and scale, as well as letting us see how they perform at different significance regimes.

We can see our results of our threshold dataset in Figure 1 and sub-threshold dataset in Figure 2 We see that in the case of highly significant individual events with the threshold dataset, statistics with the additional complexity of individual event significance have much more sensitivity, especially in the case of equation (4). This is likely because the detection Bayes factors $B_{n / s}\left(x_{a}\right)$ are more informative here due to largely separated individual real and noise populations, consistent with the observations in Piotrzkowski et al. (2021). Furthermore, we also see this same stratification in the sub-threshold dataset to a lesser effect since the detection Bayes factors are less informative. We see that the joint FAR from equation (5) performs the best in the sub-threshold case, although it's not clear whether this is due to the peculiarities with how we calculated $F A R_{g w}$. Worth noting is that like equation (4), equation (5) 
uses the significance of the GW candidate but weights this much more highly, meaning that even in this sub-threshold data there may be more of a difference between our real and noise GW datasets than expected. Regardless, we should be wary of the results involving equation (5) until this is better understood.

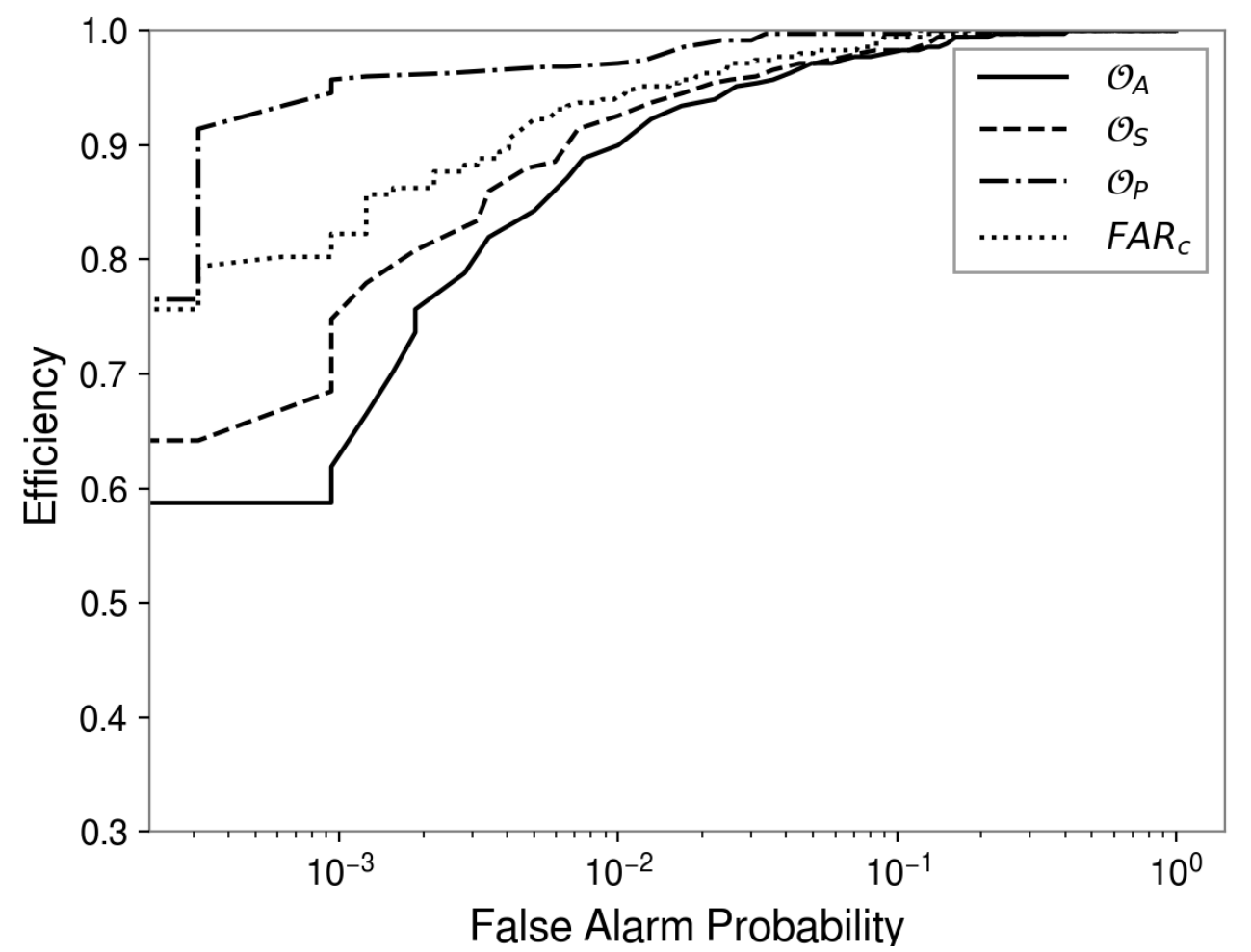

Figure 1: Simulation results for the threshold dataset described in section 2.2. testing the statistics from section 2.1 The horizontal axis is the fraction of random coincidences above a given statistic value while the vertical axis is the fraction of real coincidences above this value. We see that the additional terms in Piotrzkowski et al. (2021) $\left(\mathcal{O}_{P}\right)$ and Stachie et al. (2020) $\left(\mathcal{O}_{S}\right)$ using the individual significance of events resulted in higher sensitivity, especially for $\mathcal{O}_{P}$, compared to Ashton et al. (2018) $\left(\mathcal{O}_{A}\right)$ which does not include these. We note that higher false alarm probabilities correspond to higher efficiencies in finding real events due to the fact that the threshold to consider events of both type is lower. This is why GW searches usually have higher thresholds: although the number of real detected events is low, the number of false positives is also low in order to have high confidence in the events that do pass.

\section{Discussion}

In this work we created a simulated multi-messenger search between GWs from the LVK collaboration and GRBs from Fermi-GBM, the purpose being to test various joint ranking statistics in section 2.1. We described the nature of this simulation and the various methods to produce the necessary data products. Then we discussed the results for both a threshold and sub-threshold dataset, where we showed some evidence that statistics including the significance of the individual candidates can give more sensitivity to searches.

A major criticism of this work is the fundamental data used and the methods used to handle this data. As opposed to creating events while using LVK flavored Gaussian noise, a much more realistic GW simulation would be to do an injection campaign with real LVK noise, returning both known real injected events as well as noise transients to create the background. This will ultimately mean using robust search pipeline such as GstLAL (Sachdev et al., 2019; Cannon et al. 2021). This also would give us a more realistic $F A R_{g w}$ since this is a natural by-product for such a method. Using a GW search with real noise would better simulate realistic GW search conditions and give more weight to any conclusion made with that data. 


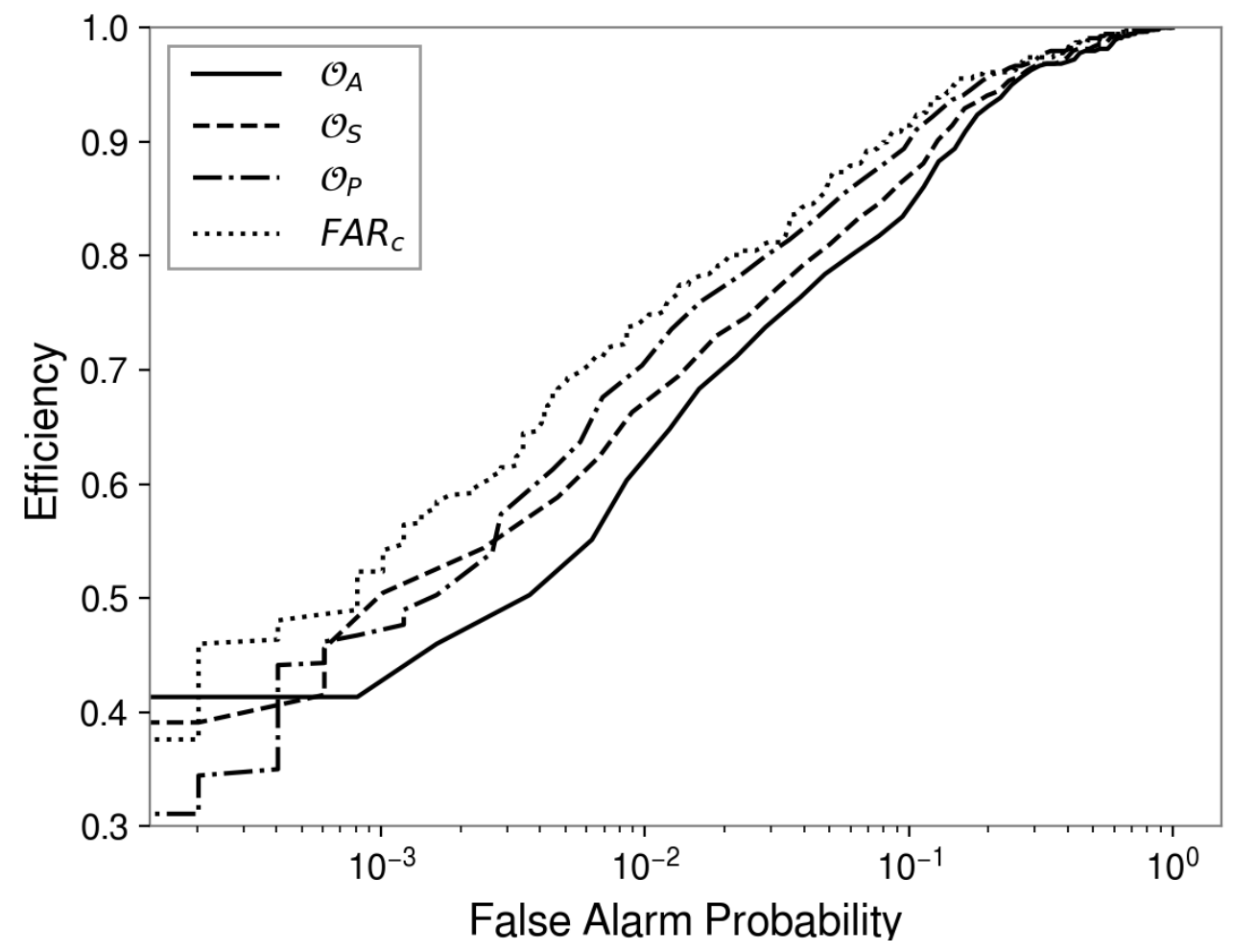

Figure 2: Simulation results for the sub-threshold dataset described in section 2.2, testing the statistics from section 2.1 similar to Figure 1 The horizontal axis is the fraction of random coincidences above a given statistic value while the vertical axis is the fraction of real coincidences above this value. We see that the additional terms in Piotrzkowski et al. (2021) $\left(\mathcal{O}_{P}\right)$ compared to Ashton et al. (2018) $\left(\mathcal{O}_{A}\right)$ and Stachie et al. (2020) $\left(\mathcal{O}_{S}\right)$ still resulted in higher sensitivity at lower significance but this reversed at the highest significance. We also see that $F A R_{c}$ from equation (5) performed the best at nearly all regimes but it's not clear whether this is due to the peculiarities of the calculation of $F A R_{g w}$ in section 2.2

The results in this study are also limited by the fact that they would not apply to a different GRB experiment such as Swift, which has a much smaller localization area and therefore a different sky map overlap distribution with the LVK (Gehrels et al. 2004). Since we omitted the additional overlap terms of distance and inclination introduced in prior statistics (Piotrzkowski et al. 2021; Ashton et al. 2020), we cannot give any indication of whether these increase sensitivity. Worth noting is that while equation (4) and (2) seem to give more sensitivity than equation (1), the need of a detection Bayes factor that requires KDEs and therefore all the events after an observing run means these aren't as helpful in low-latency searches unless a different method to approximate a detection Bayes factor is used.

As mentioned in section 2.2 the inability to completely produce a set of coherent GW and GRBs just from a set of injection parameters is currently a flaw in simulating joint BNS-GRB events. Unfortunately this will require better modeling and future constraints, both helped for the most part by more multi-messenger detections. For instance, just the relationship between viewing angle and a short GRB's luminosity still requires numerous more detections to strongly constrain this and rule out models (Farah et al. 2020). Regardless, more observations will continue to grow the field of multi-messenger astronomy and further constrain fundamental physics.

\section{Acknowledgements}

We thank Naresh Adhikari, Joshua Wood, Ignacio Magaña-Hernandez, Eric Burns, and Tito Dal Canton for useful comments and discussion which improved this work. This work makes use of the scipy (Virtanen et al., 2020), numpy (Oliphant, 2006, Van Der Walt et al., 2011; Harris et al., 2020), astropy (Robitaille et al., 2013, Price- 
Whelan et al. 2018), ligo.skymap (https:// lscsoft.docs.ligo.org/ligo.skymap/), and gwcelery (GWcelery documentation, 2021) software packages.

This work was supported by NSF award PHY-1912649. The author is grateful for computational resources provided by the LIGO Laboratory and supported by National Science Foundation Grants PHY-0757058 and PHY-0823459, and those provided by the Leonard E Parker Center for Gravitation, Cosmology and Astrophysics at the University of Wisconsin-Milwaukee. This material is based upon work supported by NASA under Award No. RFP21 6.0 issued through the Wisconsin Space Grant Consortium and the National Space Grant College and Fellowship Program.

\section{References}

Aasi, J., Abbott, B., Abbott, R., Abbott, T., Abernathy, M. R., Acernese, F., ... others (2014). Methods and results of a search for gravitational waves associated with gamma-ray bursts using the geo 600, ligo, and virgo detectors. Physical Review D, 89(12), 122004.

Abbott, B., Abbott, R., Abbott, T., Acernese, F., Ackley, K., Adams, C., ... others (2018). Gw170817: Measurements of neutron star radii and equation of state. Physical review letters, 121(16), 161101.

Abbott, B. P., Abbott, R., Abbott, T., Abernathy, M., Acernese, F., Ackley, K., ... others (2017). Search for gravitational waves associated with gamma-ray bursts during the first advanced ligo observing run and implications for the origin of grb 150906b. The Astrophysical Journal, 841(2), 89.

Abbott, B. P., Abbott, R., Abbott, T., Acernese, F., Ackley, K., Adams, C., ... others (2017a). Gravitational waves and gamma-rays from a binary neutron star merger: Gw170817 and grb 170817a. The Astrophysical Journal Letters, $848(2)$, L13.

Abbott, B. P., Abbott, R., Abbott, T., Acernese, F., Ackley, K., Adams, C., ... others (2017b). Gw170817: observation of gravitational waves from a binary neutron star inspiral. Physical Review Letters, 119(16), 161101.

Abbott, R., Abbott, T., Abraham, S., Acernese, F., Ackley, K., Adams, C., ... others (2020). Search for gravitational waves associated with gamma-ray bursts detected by fermi and swift during the ligo-virgo run o3a. arXiv preprint arXiv:2010.14550.

Ashton, G., Ackley, K., Hernandez, I. M., \& Piotrzkowski, B. (2020). Current observations are insufficient to confidently associate the binary black hole merger gw190521 with agn j124942. 3+ 344929. arXiv preprint arXiv:2009.12346.

Ashton, G., Burns, E., Dal Canton, T., Dent, T., Eggenstein, H.-B., Nielsen, A. B., .. Zhu, S. J. (2018). Coincident detection significance in multimessenger astronomy. The Astrophysical Journal, 860(1), 6.

Blinnikov, S., Novikov, I., Perevodchikova, T., \& Polnarev, A. (1984). Exploding neutron stars in close binaries. Soviet Astronomy Letters, 10, 177-179.

Cannon, K., Caudill, S., Chan, C., Cousins, B., Creighton, J. D., Ewing, B., ... others (2021). Gstlal: A software framework for gravitational wave discovery. SoftwareX, 14, 100680.

Cho, M.-A. (2019). Low-latency searches for gravitational waves and their electromagnetic counterparts with advanced ligo and virgo (Unpublished doctoral dissertation). University of Maryland.

Collaboration, L. S., Collaboration, V., Collaboration, M., Collaboration, D. E. C. G.-E., Collaboration, D., Collaboration, D., ... others (2017). A gravitational-wave standard siren measurement of the hubble constant. Nature, $551(7678), 85-88$.

Connaughton, V., Briggs, M., Goldstein, A., Meegan, C., Paciesas, W., Preece, R., .. others (2015). Localization of gamma-ray bursts using the fermi gamma-ray burst monitor. The Astrophysical Journal Supplement Series, 216(2), 32 .

Connaughton, V., Burns, E., Goldstein, A., Blackburn, L., Briggs, M., Zhang, B.-B., ... others (2016). Fermi gbm observations of ligo gravitational-wave event gw150914. The Astrophysical Journal Letters, 826(1), L6. 
Coughlin, M. W., Dietrich, T., Margalit, B., \& Metzger, B. D. (2019). Multimessenger bayesian parameter inference of a binary neutron star merger. Monthly Notices of the Royal Astronomical Society: Letters, 489(1), L91-L96.

Eichler, D., Livio, M., Piran, T., \& Schramm, D. N. (1989). Nucleosynthesis, neutrino bursts and $\gamma$-rays from coalescing neutron stars. Nature, 340(6229), 126-128.

Farah, A., Essick, R., Doctor, Z., Fishbach, M., \& Holz, D. E. (2020). Counting on short gamma-ray bursts: Gravitational-wave constraints of jet geometry. The Astrophysical Journal, 895(2), 108.

Gehrels, N., Chincarini, G., Giommi, P., Mason, K., Nousek, J. A., Wells, A., .. others (2004). The swift gamma-ray burst mission. The Astrophysical Journal, 611(2), 1005.

Goldstein, A., Hamburg, R., Wood, J., Hui, C. M., Cleveland, W. H., Kocevski, D., ... others (2019). Updates to the fermi gbm targeted sub-threshold search in preparation for the third observing run of ligo/virgo. arXiv preprint arXiv:1903.12597.

Goldstein, A., Veres, P., Burns, E., Briggs, M., Hamburg, R., Kocevski, D., .. others (2017). An ordinary short gamma-ray burst with extraordinary implications: Fermi-gbm detection of grb 170817a. The Astrophysical Journal Letters, 848(2), L14.

Graham, M., Ford, K., McKernan, B., Ross, N., Stern, D., Burdge, K., .. o others (2020). Candidate electromagnetic counterpart to the binary black hole merger gravitational-wave event s190521g. Physical review letters, 124(25), 251102.

Greiner, J., Burgess, J. M., Savchenko, V., \& Yu, H.-F. (2016). On the fermi-gbm event 0.4 s after gw150914. The Astrophysical Journal Letters, 827(2), L38.

Gwcelery documentation. (2021). https://igwn.readthedocs.io/projects/gwcelery/en/ latest/gwcelery.conf.html, (accessed 01-June-2021)

Hamburg, R., Fletcher, C., Burns, E., Goldstein, A., Bissaldi, E., Briggs, M., .. others (2020). A joint fermi-gbm and ligo/virgo analysis of compact binary mergers from the first and second gravitational-wave observing runs. The Astrophysical Journal, 893(2), 100.

Harris, C. R., Millman, K. J., van der Walt, S. J., Gommers, R., Virtanen, P., Cournapeau, D., .. others (2020). Array programming with numpy. Nature, 585(7825), 357-362.

Howell, E., Ackley, K., Rowlinson, A., \& Coward, D. (2019). Joint gravitational wave-gamma-ray burst detection rates in the aftermath of gw170817. Monthly Notices of the Royal Astronomical Society, 485(1), $1435-1447$.

Nitz, A. H., Nielsen, A. B., \& Capano, C. D. (2019). Potential gravitational-wave and gamma-ray multi-messenger candidate from oct. 30, 2015. arXiv preprint arXiv:1902.09496.

Oliphant, T. E. (2006). A guide to NumPy (Vol. 1). Trelgol Publishing USA.

Paczynski, B. (1986). Gamma-ray bursters at cosmological distances. The Astrophysical Journal, 308, L43-L46.

Piotrzkowski, B. J., Baylor, A., \& Magaña-Hernandez, I. (2021). A joint ranking statistic for multi-messenger astronomical searches with gravitational waves. In prep.

Price-Whelan, A. M., Sipőcz, B., Günther, H., Lim, P., Crawford, S., Conseil, S., .. others (2018). The astropy project: Building an open-science project and status of the v2. 0 core package. The Astronomical Journal, 156(3), 123.

Robitaille, T. P., Tollerud, E. J., Greenfield, P., Droettboom, M., Bray, E., Aldcroft, T., ... others (2013). Astropy: A community python package for astronomy. Astronomy \& Astrophysics, 558, A33.

Sachdev, S., Caudill, S., Fong, H., Lo, R. K., Messick, C., Mukherjee, D., .. others (2019). The gstlal search analysis methods for compact binary mergers in advanced ligo's second and advanced virgo's first observing runs. arXiv preprint arXiv:1901.08580. 
Singer, L. P., Price, L. R., Farr, B., Urban, A. L., Pankow, C., Vitale, S., .. others (2014). The first two years of electromagnetic follow-up with advanced ligo and virgo. The Astrophysical Journal, 795(2), 105.

Stachie, C., Canton, T. D., Burns, E., Christensen, N., Hamburg, R., Briggs, M., ... others (2020). Search for advanced ligo single interferometer compact binary coalescence signals in coincidence with gamma-ray events in fermi-gbm. arXiv preprint arXiv:2001.01462.

Urban, A. L. (2016). Monsters in the dark: High energy signatures of black hole formation with multimessenger astronomy (Unpublished doctoral dissertation). University of Wisconsin-Milwaukee.

Van Der Walt, S., Colbert, S. C., \& Varoquaux, G. (2011). The numpy array: a structure for efficient numerical computation. Computing in Science \& Engineering, 13(2), 22.

Virtanen, P., Gommers, R., Oliphant, T. E., Haberland, M., Reddy, T., Cournapeau, D., ... Contributors, S. . . (2020). SciPy 1.0: Fundamental Algorithms for Scientific Computing in Python. Nature Methods, 17, 261-272. doi: https://doi.org/10.1038/s41592-019-0686-2 